\title{
Enhanced Expression of the S100A8/A9 Complex in Acute Myocardial Infarction Patients
}

\author{
Takashi Katashima, MD; Takahiko Naruko, MD*; Fumio Terasaki, MD; Masatoshi Fujita, MD**; \\ Kaoru Otsuka, MD; Shougo Murakami, MD; Akira Sato, MD ${ }^{\dagger}$; Michiaki Hiroe, MD ${ }^{\dagger}$; \\ Yoshihiro Ikura, MD*; Makiko Ueda, MD*; Masaki Ikemoto, PhD**; Yasushi Kitaura, MD
}

\begin{abstract}
Background: S100A8/A9 complex (S100A8/A9) is expressed in activated human neutrophils and macrophages. Enhanced expression of S100A8/A9 in atherosclerotic plaque of patients with unstable angina pectoris (UAP) has been demonstrated, but its profile in acute myocardial infarction (AMI) has not been clarified.

Methods and Results: Serum S100A8/A9 levels were serially measured in patients with AMI $(n=55)$ and UAP $(n=16)$ during the acute period. The expression of S100A8/A9 was examined immunohistochemically in the infarcted myocardium of 7 autopsied patients with AMI. Serum S100A8/A9 levels on the 1st day were 1,118 \pm 115 (SE) $\mathrm{ng} / \mathrm{ml}$ in AMI patients as compared with $787 \pm 147 \mathrm{ng} / \mathrm{ml}$ in UAP patients. On days $3-5$, serum S100A8/A9 levels in AMI patients reached a peak value and were significantly higher than the values in UAP patients $(1,690 \pm 144 \mathrm{ng} / \mathrm{ml}$ vs $844 \pm 100 \mathrm{ng} / \mathrm{ml} ; \mathrm{P}<0.0001)$. In AMI patients, peak S100A8/A9 levels positively correlated with peak white blood cell and neutrophil counts, and peak creatine kinase-MB and peak C-reactive protein levels. Double immunostaining revealed that S100A8/A9 was specifically expressed in neutrophils and macrophages infiltrating the infarcted myocardium.
\end{abstract}

Conclusions: S100A8/A9 is implicated in the pathophysiology of AMI and may be an additional biomarker of the local inflammatory response following AMI. (Circ J 2010; 74: 741-748)

Key Words: Inflammation; Myocardial infarction; Pathology; Proteins

$\mathbf{T}$ he S100A8/A9 complex is a heterodimeric molecule of the S100 family and is composed of S100A8 $(10.8 \mathrm{kD})$ and S100A9 $(13.2 \mathrm{kD})$, containing 2 calcium-binding sites per molecule. The 2 subunits are expressed in activated human neutrophils and macrophages in inflammatory lesions. S100A8 and S100A9 noncovalently associate to quickly form a heterodimer, the S100A8/A9 complex. ${ }^{1}$ Translocation of the complex from the cytosol to the membrane is induced by activation of a novel pathway requiring an intact microtubule network. ${ }^{2}$

\section{Editorial p 626}

Recent clinical studies have demonstrated elevated serum levels of the S100A8/A9 complex in inflammatory disorders, such as rheumatoid arthritis, ${ }^{3}$ transplant rejection, ${ }^{4}$ unstable angina pectoris (UAP), ${ }^{5}$ acute myocardial infarction (AMI), ${ }^{6,7}$ and recent MI. ${ }^{8}$ Overexpression of the S100A8/A9 complex has been shown immunohistochemically in atherosclerotic plaques in coronary and carotid arteries. ${ }^{5,6,9}$ High levels of S100A9 in human atherosclerotic rupture-prone lesions are also reported. ${ }^{10}$ Furthermore, the mechanistic implication of S100A8/A9 in the pathophysiology of atherosclerosis and vascular inflammation has been demonstrated. ${ }^{11}$ Thus, elevated serum S100A8/A9 levels in AMI are attributable to coronary vulnerable plaques.

Meanwhile, it has been reported that activated macrophages infiltrating the myocardial tissue of patients with active cardiac sarcoidosis highly express the S100A8/A9 complex. ${ }^{12}$ Although myocardial necrosis is the major histological feature of AMI, it is a well-known fact that inflammatory responses develop in the infarcted myocardium. ${ }^{13}$ Neutrophil infiltration is present at the border of the infarct $24 \mathrm{~h}$ after symptom onset, and macrophages begin to appear

Received August 3, 2009; revised manuscript received December 19, 2009; accepted December 21, 2009; released online February 27, 2010 Time for primary review: 29 days

Department of Internal Medicine III, Osaka Medical College, Takatsuki, *Department of Cardiology, Osaka City General Hospital, Osaka, **Human Health Sciences, Kyoto University Graduate School of Medicine, Kyoto, 'Department of Cardiology, University of Tsukuba Graduate School of Comprehensive Human Science, Tsukuba, ${ }^{\dagger}$ Department of Cardiology, International Medical Center of Japan, Tokyo and Department of Pathology, Osaka City University Graduate School of Medicine, Osaka, Japan

The first two authors contributed equally to this work.

Mailing address: Fumio Terasaki, MD, Department of Internal Medicine III, Osaka Medical College, 2-7 Daigaku-machi, Takatsuki 569-8686, Japan. E-mail: in3012@ poh.osaka-med.ac.jp

ISSN-1346-9843 doi:10.1253/circj.CJ-09-0564

All rights are reserved to the Japanese Circulation Society. For permissions, please e-mail: cj@j-circ.or.jp 


\begin{tabular}{|c|c|c|c|}
\hline Characteristics & AMI $(n=55)$ & UAP $(n=16)$ & $P$ value \\
\hline Age (years) & $66.8 \pm 1.4$ & $74.8 \pm 1.8$ & 0.005 \\
\hline $\operatorname{Sex}(M / F)$ & $37 / 18$ & $10 / 6$ & NS \\
\hline \multicolumn{4}{|l|}{ Risk factors } \\
\hline Hypertension, n (\%) & $39(71)$ & $12(75)$ & NS \\
\hline $\mathrm{SBP}(\mathrm{mmHg})$ & $144.4 \pm 3.5$ & $152.8 \pm 5.6$ & NS \\
\hline $\mathrm{DBP}(\mathrm{mmHg})$ & $86.9 \pm 2.3$ & $82.0 \pm 2.6$ & NS \\
\hline $\mathrm{DM}, \mathrm{n}(\%)$ & $29(53)$ & $6(38)$ & NS \\
\hline FBS (mg/dl) & $115.0 \pm 3.6$ & $106.2 \pm 3.5$ & NS \\
\hline Hypercholesterolemia, n (\%) & $38(69)$ & $12(75)$ & NS \\
\hline LDL-cholesterol (mg/dl) & $136.6 \pm 5.6$ & $135.4 \pm 8.6$ & NS \\
\hline HDL-cholesterol (mg/dl) & $51.2 \pm 1.9$ & $47.1 \pm 2.2$ & NS \\
\hline Triglyceride $(\mathrm{mg} / \mathrm{dl})$ & $136.0 \pm 10.6$ & $151.8 \pm 19.0$ & NS \\
\hline Smoking, $\mathrm{n}(\%)$ & $12(22)$ & $3(19)$ & NS \\
\hline $\mathrm{BMI}\left(\mathrm{kg} / \mathrm{m}^{2}\right)$ & $23.6 \pm 0.4$ & $22.5 \pm 0.3$ & NS \\
\hline \multicolumn{4}{|l|}{ Prior medication } \\
\hline Aspirin, n (\%) & $29(49)$ & $12(75)$ & NS \\
\hline$\beta$-blocker, $\mathrm{n}(\%)$ & $12(22)$ & $5(31)$ & NS \\
\hline ACE inhibitor/ARB, n (\%) & $31(56)$ & $10(63)$ & NS \\
\hline Calcium channel blocker, $\mathrm{n}(\%)$ & $19(35)$ & $9(56)$ & NS \\
\hline Nitrate, $n(\%)$ & $16(29)$ & $6(38)$ & NS \\
\hline Statin, n (\%) & $25(45)$ & $6(38)$ & NS \\
\hline \multicolumn{4}{|l|}{ Laboratory data } \\
\hline WBC $\left(\times 10^{3} / \mu l\right)$ & $8.5 \pm 0.4$ & $6.8 \pm 0.3$ & 0.017 \\
\hline Neutrophils $\left(\times 10^{3} / \mu l\right)$ & $6.2 \pm 0.4$ & $4.5 \pm 0.3$ & 0.020 \\
\hline CK-MB (U/L) & $44.0 \pm 6.9$ & $14.2 \pm 2.0$ & NS \\
\hline CRP (mg/dl) & $5.6 \pm 1.6$ & $2.2 \pm 0.8$ & NS \\
\hline \multicolumn{4}{|l|}{ Angiographic findings } \\
\hline 1-vessel disease, $\mathrm{n}(\%)$ & $25(45)$ & $2(12)$ & NS \\
\hline 2-vessel disease, $\mathrm{n}(\%)$ & $21(38)$ & $8(50)$ & NS \\
\hline 3-vessel disease, $\mathrm{n}(\%)$ & $9(16)$ & $6(38)$ & NS \\
\hline Time from onset of chest pain to first blood draw (h) & $4.7 \pm 0.5$ & $4.7 \pm 1.1$ & NS \\
\hline Time from onset of chest pain to $\mathrm{PCl}(\mathrm{h})$ & $6.0 \pm 0.5$ & $7.7 \pm 1.1$ & NS \\
\hline
\end{tabular}

Values are expressed as the mean $\pm \mathrm{SE}$.

$\mathrm{AMI}$, acute myocardial infarction; UAP, unstable angina pectoris; NS, not significant; SBP, systolic blood pressure; DBP, diastolic blood pressure; DM, diabetes mellitus; FBS, fasting blood sugar; LDL, low-density lipoprotein; HDL, high-density lipoprotein; BMI, body mass index; ACE, angiotensin-converting enzyme; ARB, angiotensin receptor blocker; WBC, white blood cell; CK-MB, creatine kinase isoenzyme MB fraction; CRP, C-reactive protein; PCl, percutaneous coronary intervention.

in the infarct area 3-5 days after symptom onset. By 1 week after symptom onset, neutrophils have decreased and granulation tissue becomes established with neocapillary invasion, and infiltration of macrophages, lymphocytes, and plasma cells. ${ }^{14}$ Therefore, an alternative source of the S100A8/A9 complex may be these inflammatory cells. We hypothesized that the source of the S100A8/A9 complex in AMI may not only be coronary atherosclerotic plaques, but also the infarcted myocardium. Therefore, the purpose of this study was to compare serially measured serum S100A8/A9 levels of patients with AMI, and those of patients with UAP, as well as immunohistochemically examining the expression profile of the S100A8/A9 complex in myocardium obtained from autopsied AMI patients.

\section{Methods}

\section{Clinical Studies}

The study cohort consisted of 71 patients with either AMI

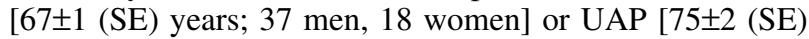

years; 10 men, 6 women]. AMI was diagnosed on the basis of: (1) chest pain $>30 \mathrm{~min}$ in duration, (2) ST-segment elevation $>0.1 \mathrm{mV}$ in 2 contiguous ECG leads, (3) total or subtotal occlusion of the infarct-related artery, (4) elevated creatine kinase (CK)-MB isoenzyme more than 2 -fold within $12 \mathrm{~h}$ of chest pain, and (5) successful primary coronary angioplasty (defined as Thrombolysis In Myocardial Infarction flow ${ }^{15}$ grade 3 with a residual diameter stenosis $<30 \%$ ). UAP was diagnosed according to Braunwald's criteria. ${ }^{16}$ We excluded patients with non-atherosclerotic inflammatory disorders, such as pneumonia and vasculitis, in order to avoid potentially confounding effects with respect to serum levels of the S100A8/A9 complex. A total of 30 healthy volunteers who were free of chronic conditions associated with coronary artery disease, such as hyperlipidemia, hypertension and diabetes, served as controls.

This study complied with the Declaration of Helsinki, and was approved by the institutional review committees of the Osaka Medical College Hospital; the subjects gave informed consent. 


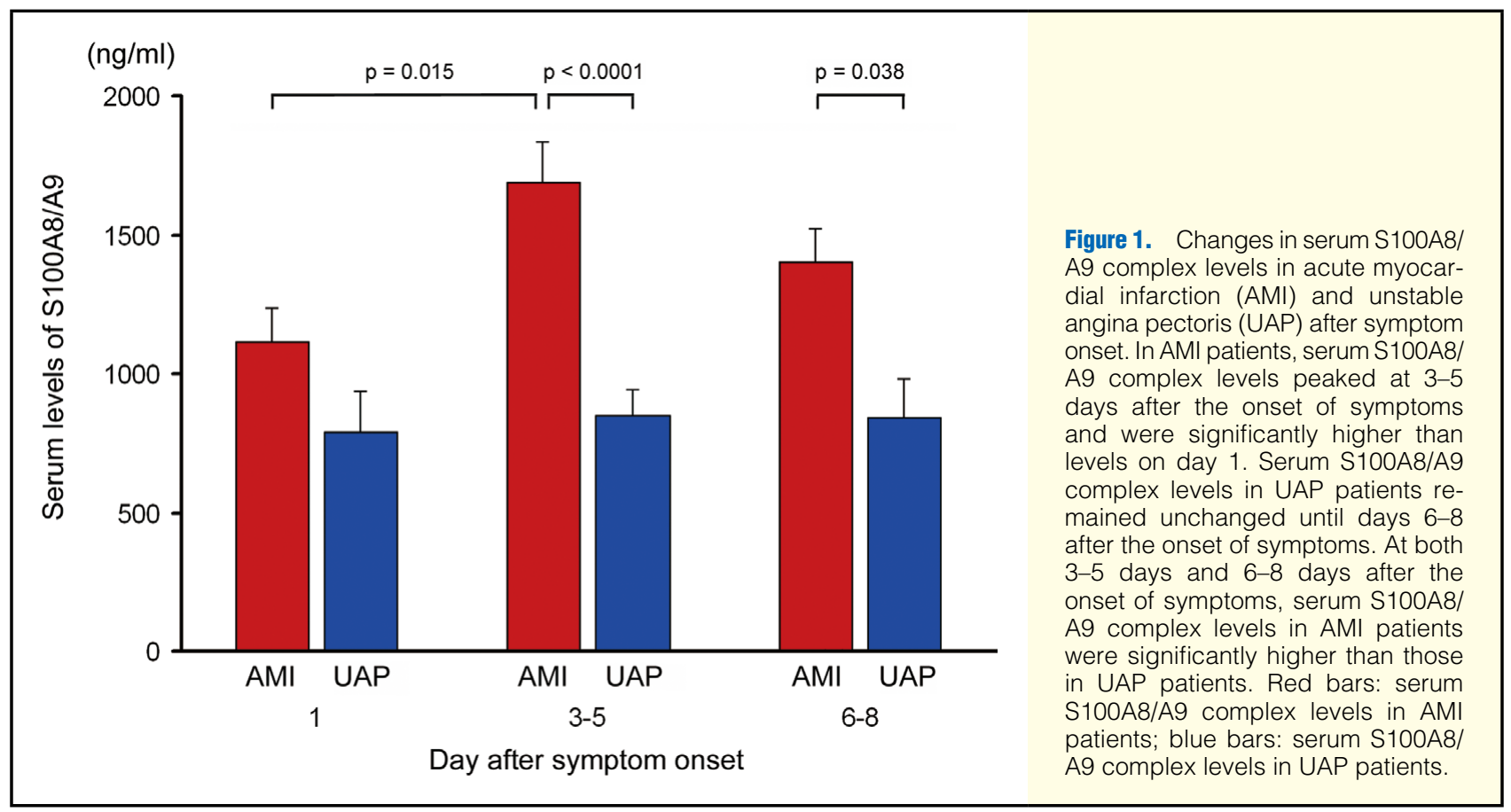

Measurement of Serum Levels of the S100A8/A9 Complex

Serum S100A8/A9 complex levels were serially measured, using a sandwich enzyme-linked immunosorbent assay system that was developed in our laboratory, ${ }^{4}$ in patients with AMI and UAP during the acute period [ie, from the time of symptom onset (day 1 of hospitalization) to day 8 of hospitalization]. All AMI and UAP patients underwent successful primary coronary angioplasty within $12 \mathrm{~h}$ of the onset of symptoms. The first sample was taken prior to revascularization treatment and subsequent samples were taken after treatment in all patients. White blood cell (WBC) counts and serum levels of C-reactive protein (CRP), CK, and CK-MB were measured by conventional methods. Comparison analyses were conducted between serum S100A8/A9 complex levels and other clinical features.

\section{Pathological Studies}

Frozen myocardial samples were obtained at autopsy from 7 patients who had died between 1 and 9 days after the onset of AMI. In addition, frozen myocardial samples from individuals who died of non-cardiovascular causes $(n=6)$ served as references. The frozen samples were subsequently serially sectioned at a thickness of $6 \mu \mathrm{m}$, and fixed in acetone. All first sections were stained with hematoxylin-eosin; the other sections were used for immunohistochemical staining.

\section{Immunohistochemistry}

Single Staining The cellular components were analyzed using monoclonal antibodies against macrophages (EBM11; DAKO, Glostrup, Denmark) and neutrophils (CD66b; Beckman Coulter, Fullerton, CA, USA). Commercially available monoclonal antibodies against S100A8 and S100A9 (both from Santa Cruz Biotechnology, Santa Cruz, CA, USA) and a rabbit polyclonal antibody against the S100A8/A9 complex were used to investigate immunolocalization of these protein monomers and complex. The methods of the antiS100A8/A9 complex antibody production and specificity testing were reported previously. ${ }^{4}$ The specificity of the results

\begin{tabular}{|lcc|}
\hline $\begin{array}{l}\text { Table 2. Correlation of Peak Serum S100A8/A9 Complex } \\
\text { Levels With Laboratory Variables in Patients } \\
\text { With AMI }\end{array}$ & \multicolumn{3}{c|}{ r } & P value \\
& 0.453 & 0.001 \\
Peak WBC $\left(\times 10^{3} / \mu \mathrm{l}\right)$ & 0.444 & 0.002 \\
Peak neutrophils $\left(\times 10^{3} / \mu \mathrm{l}\right)$ & 0.307 & 0.036 \\
Peak CK-MB $(\mathrm{U} / \mathrm{L})$ & 0.370 & 0.011 \\
Peak CRP $(\mathrm{mg} / \mathrm{dl})$ & & \\
\hline
\end{tabular}

Abbreviations see in Table 1.

obtained with these antibodies was checked by omitting the primary antibodies and using non-immune mouse IgG serum (DAKO) and non-immune rabbit serum (DAKO) for monoclonal and polyclonal antibodies, respectively, as negative controls. Sections were incubated at $4^{\circ} \mathrm{C}$ overnight or $1 \mathrm{~h}$ at room temperature, and then subjected to a 3-step staining procedure, using the streptavidin-biotin complex method for detection. Peroxidase activity was visualized with 3-amino-9ethyl-carbazole (10 min, room temperature), and the sections were faintly counterstained with hematoxylin.

Double Immunostaining To identify cell types that stained positive for the S100A8/A9 complex, we also performed double immunostaining for the neutrophil (CD66b)/ S100A8/A9 complex and macrophage (CD68)/S100A8/A9 complex, according to the previously described method, with minor procedural modifications. ${ }^{17}$ In this staining method, alkaline phosphatase is visualized with fast blue BB (blue, neutrophils or macrophages), and peroxidase is visualized with 3-amino-9-ethyl-carbazole (red, S100A8/A9 complex).

Quantitative Methods The area occupied by S100A8-, S100A9-, and S100A8/A9-complex-positive cells was quantified by computer-aided planimetry, and expressed as a percentage of the myocardial tissue area. The number of CD66b-positive neutrophils was counted and expressed as the number of cells $/ \mathrm{mm}^{2}$ of myocardial tissue. The macrophagepositive area was quantified and expressed as percent area. 


\begin{tabular}{|c|c|c|c|c|c|}
\hline Case no. & Age/sex & IRA & $\begin{array}{l}\text { Interval between } \\
\text { symptom onset } \\
\text { and death }\end{array}$ & Risk factors & Cause of death \\
\hline 1 & 70/M & LAD & $6 \mathrm{~h}$ & Smoking & Cardiac rupture \\
\hline 2 & $73 / F$ & RCA & 1 day & HT, HL, DM, smoking & Cardiac rupture \\
\hline 3 & $58 / \mathrm{M}$ & $\mathrm{RCA}$ & 2 days & DM, smoking & CHF due to multivessel disease \\
\hline 4 & $62 / \mathrm{M}$ & LMT & 2 days & Smoking & $\mathrm{CHF}$ \\
\hline 5 & $79 / M$ & LAD & 7 days & $\mathrm{HT}$, smoking & VF \\
\hline 6 & $69 / \mathrm{M}$ & LMT & 9 days & Smoking & $\mathrm{CHF}$ \\
\hline 7 & $84 / \mathrm{M}$ & LAD & 9 days & HT & CHF due to multivessel disease \\
\hline
\end{tabular}

IRA, infarct-related artery; LAD, left anterior descending coronary artery; RCA, right coronary artery; $H T$, hypertension; $\mathrm{HL}$, hyperlipidemia; CHF, congestive heart failure; LMT, left main trunk; VF, ventricular fibrillation. Other abbreviations see in Table 1.
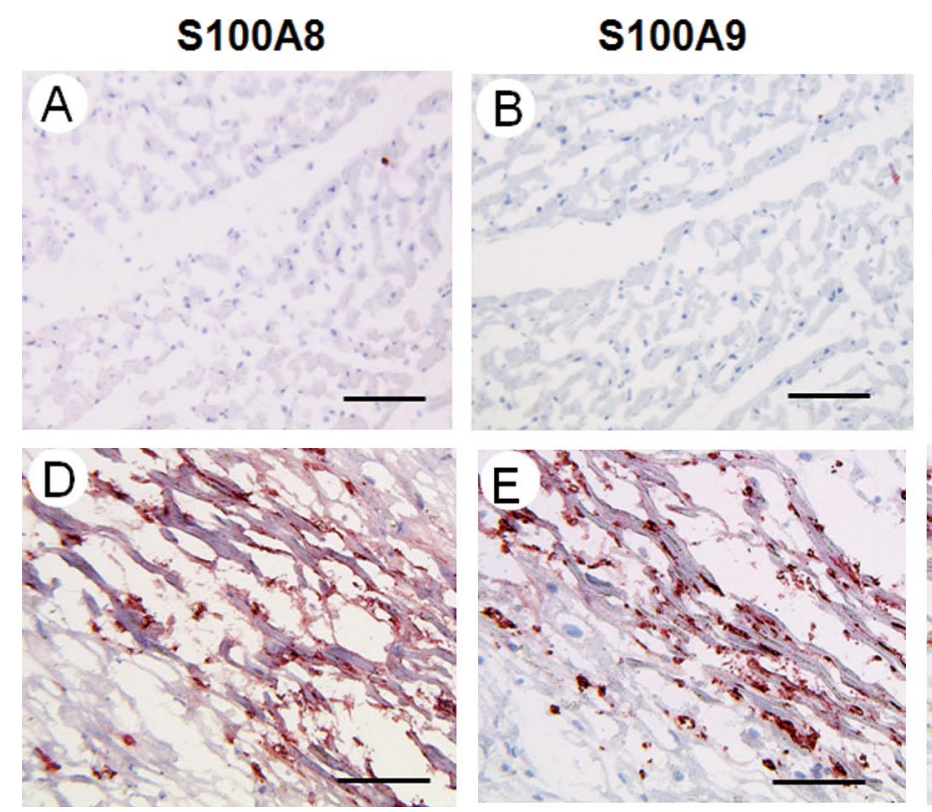

\section{S100A8/A9 Complex}
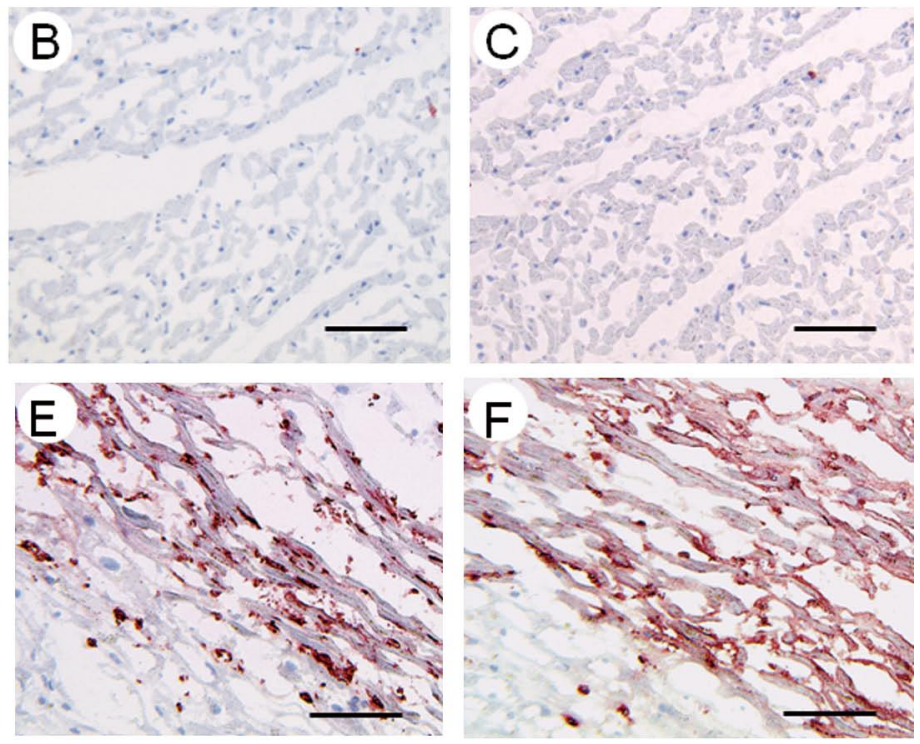

Figure 2. Micrographs showing S100A8-, S100A9-, and S100A8/A9-complex-expression in autopsied human myocardium from a reference case $(\mathbf{A}-\mathbf{C})$ and from an AMI patient (D-F). (A,D) Immunostaining for S100A8; $(\mathbf{B}, \mathbf{E})$ immunostaining for S100A9; (C,F) immunostaining for S100A8/A9 complex. The anti-S100A8, S100A9, and S100A8/A9-complex antibodies exhibit scattered S100A8-, S100A9-, S100A8/A9-complex-positive cells in the myocardium from a reference case, whereas they show abundant positive cells in the infarcted myocardium from an AMI patient (case 2 in Table 3). Bars $=100 \mu \mathrm{m}$.

In addition, on the basis of double immunostaining for the neutrophil (CD66b)/S100A8/A9 complex and macrophage (CD68)/S100A8/A9 complex, the percentage of S100A8/A9complex-positive neutrophils in the total neutrophil count and S100A8/A9-complex-positive macrophages in the total macrophage count were calculated.

\section{Statistical Analysis}

Data are presented as the mean \pm SE. Comparing parameters between 2 groups was performed with the chi-square test or the unpaired 2-tailed Student's t-test. Comparison across more than 2 groups involved 1-way analysis of variance (ANOVA) with Bonferroni correction when appropriate. Serum S100A8/ A9 complex levels were compared over time, using repeated measure ANOVA. Pearson's correlation coefficients were used to evaluate the relations between serum S100A8/A9 complex levels and clinical parameters. The morphometric analysis was performed by a single investigator who was unaware of the patients' characteristics and histological classifications. For comparisons of 2 groups of individuals, the Mann-Whitney U test was used in all circumstances. Differences were considered statistically significant at $\mathrm{P}<0.05$.

\section{Results}

\section{Clinical Information}

Clinical features of the 55 AMI and 16 UAP patients on admission are shown in Table 1. The AMI patients were younger than the UAP patients. WBC and neutrophil counts were higher in the AMI patients than in the UAP patients. Otherwise, there were no statistically significant differences between the 2 groups. 


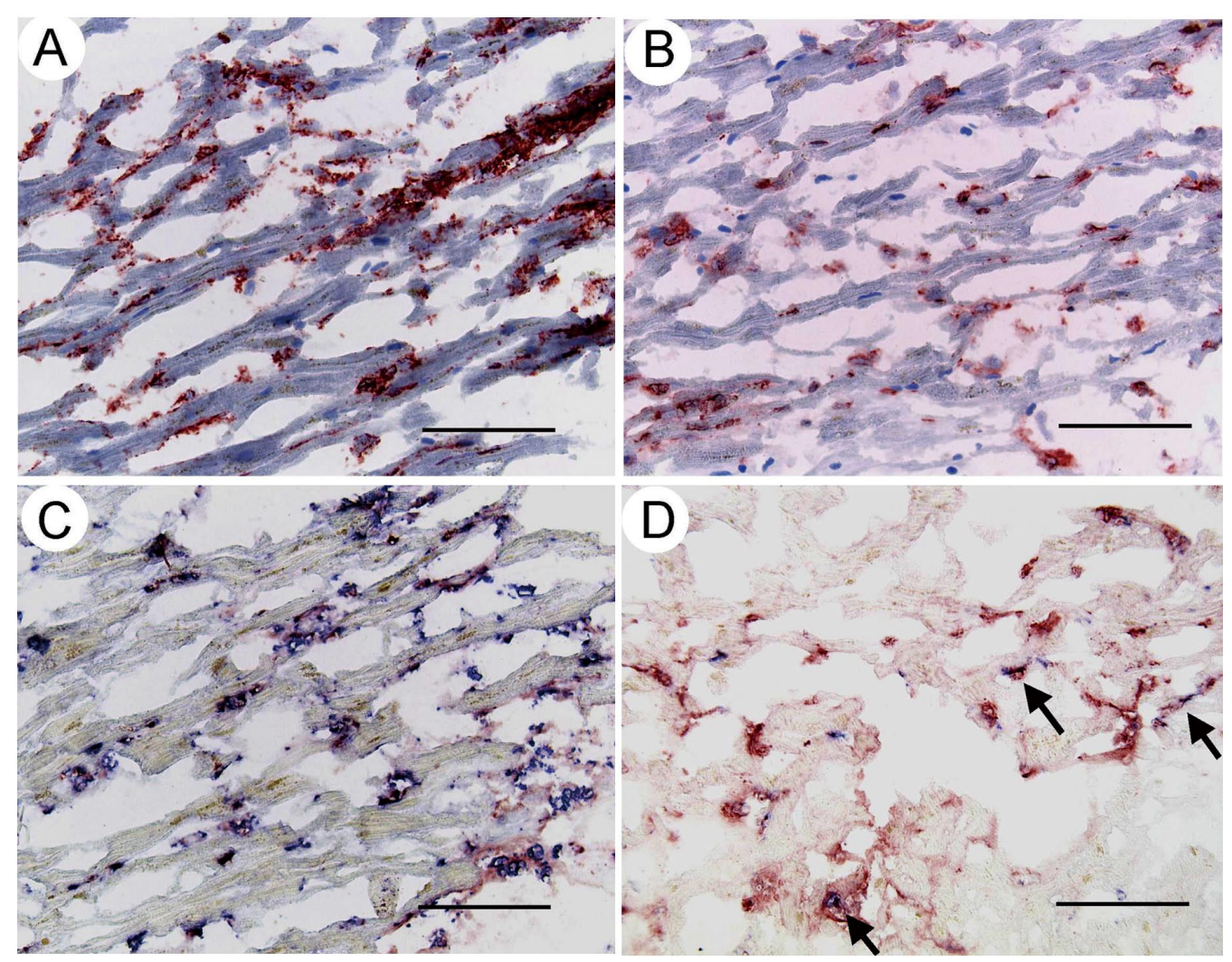

Figure 3. Micrographs of the infarcted myocardium from an autopsied patient (case 3 in Table 3). (A) Anti-CD66b antibody staining reveals a large number of neutrophils in the infarct area. (B) An adjacent section stained with anti-S100A8/A9 complex also reveals the presence of S100A8/A9-complex-positive cells. (C) Double immunostaining for CD66b (blue) and the S100A8/ A9 complex (red) reveals that most cells show double staining (purple), thus indicating that most S100A8/A9-complex-positive cells are neutrophils. (D) Double immunostaining for macrophages (blue) and the S100A8/A9 complex (red) reveals that few cells (arrows) show double staining (purple), thus indicating that few S100A8/A9-complex-positive cells are macrophages. Bars $=100 \mu \mathrm{m}$.

\begin{abstract}
Measurement of Serum Levels of the S100A8/A9 Complex The serum S100A8/A9 complex levels on day 1 of symptom onset were significantly higher in AMI patients than in normal controls $[1,118 \pm 115$ (SE) $\mathrm{ng} / \mathrm{ml}$ vs $230 \pm 21 \mathrm{ng} / \mathrm{ml}$; $\mathrm{P}<$ $0.0001]$, and peaked between days 3 and $5(1,690 \pm 144 \mathrm{ng} / \mathrm{ml})$. In UAP patients, the serum S100A8/A9 complex levels on day 1 of symptom onset were $787 \pm 147 \mathrm{ng} / \mathrm{ml}$, which was also significantly higher than that in normal controls $(\mathrm{P}=0.028)$. The serum S100A8/A9 complex levels in UAP patients remained unchanged until days 6-8 after symptom onset. On days 3-5, the serum S100A8/A9 complex levels was significantly higher in AMI patients than in UAP patients $(1,690 \pm$ $144 \mathrm{ng} / \mathrm{ml}$ vs $844 \pm 100 \mathrm{ng} / \mathrm{ml}$; P<0.0001; Figure 1). In AMI patients the peak level of S100A8/A9 complex positively correlated with the peak WBC count $(r=0.453, P=0.001)$, peak neutrophil count $(\mathrm{r}=0.444, \mathrm{P}=0.002)$, peak CK-MB level $(\mathrm{r}=0.307, \mathrm{P}=0.036)$, and peak $\mathrm{CRP}$ level $(\mathrm{r}=0.370, \mathrm{P}=0.011)$ (Table 2).
\end{abstract}

\section{Pathological Studies}

The clinical characteristics of the 7 autopsied patients with AMI are summarized in Table 3 . Regarding the staining pattern of S100A8, S100A9, and the S100A8/A9 complex, there was no significant difference among the 3 antibodies.
The anti-S100A8, S100A9, and S100A8/A9-antibodies showed scattered S100A8-, S100A9-, S100A8/A9-complexpositive cells in the myocardium from the reference cases (Figures 2A-C), whereas in the infarcted myocardium of the AMI patients the 3 antibodies showed abundant S100A8, S100A9, and S100A8/A9-complex-positive cells (Figures 2 D-F). The S100A8/A9 complex was specifically and strongly positive in neutrophils and macrophages infiltrating the infarcted myocardium of AMI patients. In the early acute phase (from $6 \mathrm{~h}$ to 2 days after the onset of AMI), neutrophils were the main component of infiltrating inflammatory cells. Double immunostaining for the S100A8/A9 complex and macrophages or neutrophils showed that the vast majority of S100A8/A9-complex-positive cells were neutrophils at this stage (Figure 3). Meanwhile, in the subacute phase (7-9 days after the onset of AMI), the number of accumulating macrophages increased, and double immunostaining showed that most S100A8/A9-complex cells were macrophages in this phase (Figure 4).

Quantitative analysis demonstrated that the S100A8-, S100A9-, and S100A8/A9-complex-positive areas were significantly larger $(\mathrm{S} 100 \mathrm{~A} 8, \mathrm{P}<0.005 ; \mathrm{S} 100 \mathrm{~A} 9, \mathrm{P}<0.005$; S100A8/A9 complex, $\mathrm{P}<0.005)$ in the infarcted myocardium of AMI patients than in the myocardium from the reference 


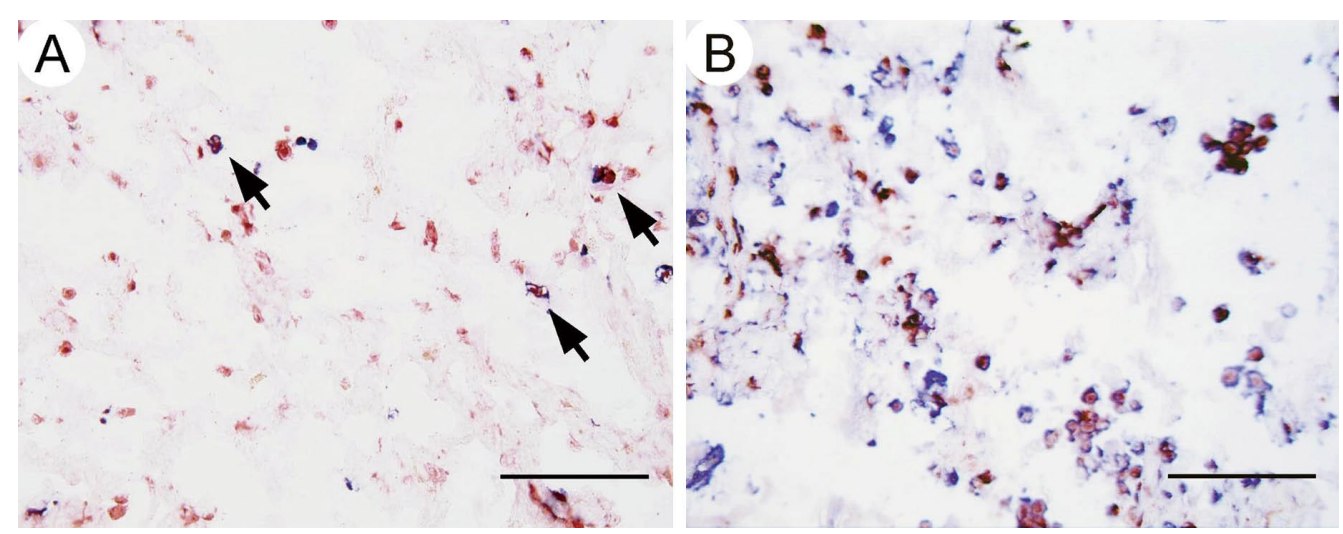

Figure 4. Micrographs of the infarcted myocardium of an autopsied patient (case 7 in Table 3). (A) Double immunostaining for neutrophils (blue) and the S100A8/A9 complex (red) reveals that few cells (arrows) show double staining (purple), thus indicating that few S100A8/A9-complex-positive cells are neutrophils. (B) Double immunostaining for CD68 (blue) and the S100A8/A9 complex (red) reveals that most cells show double staining (purple), thus indicating that, at this stage, most S100A8/A9-complex-positive cells are macrophages. Bars $=100 \mu \mathrm{m}$.

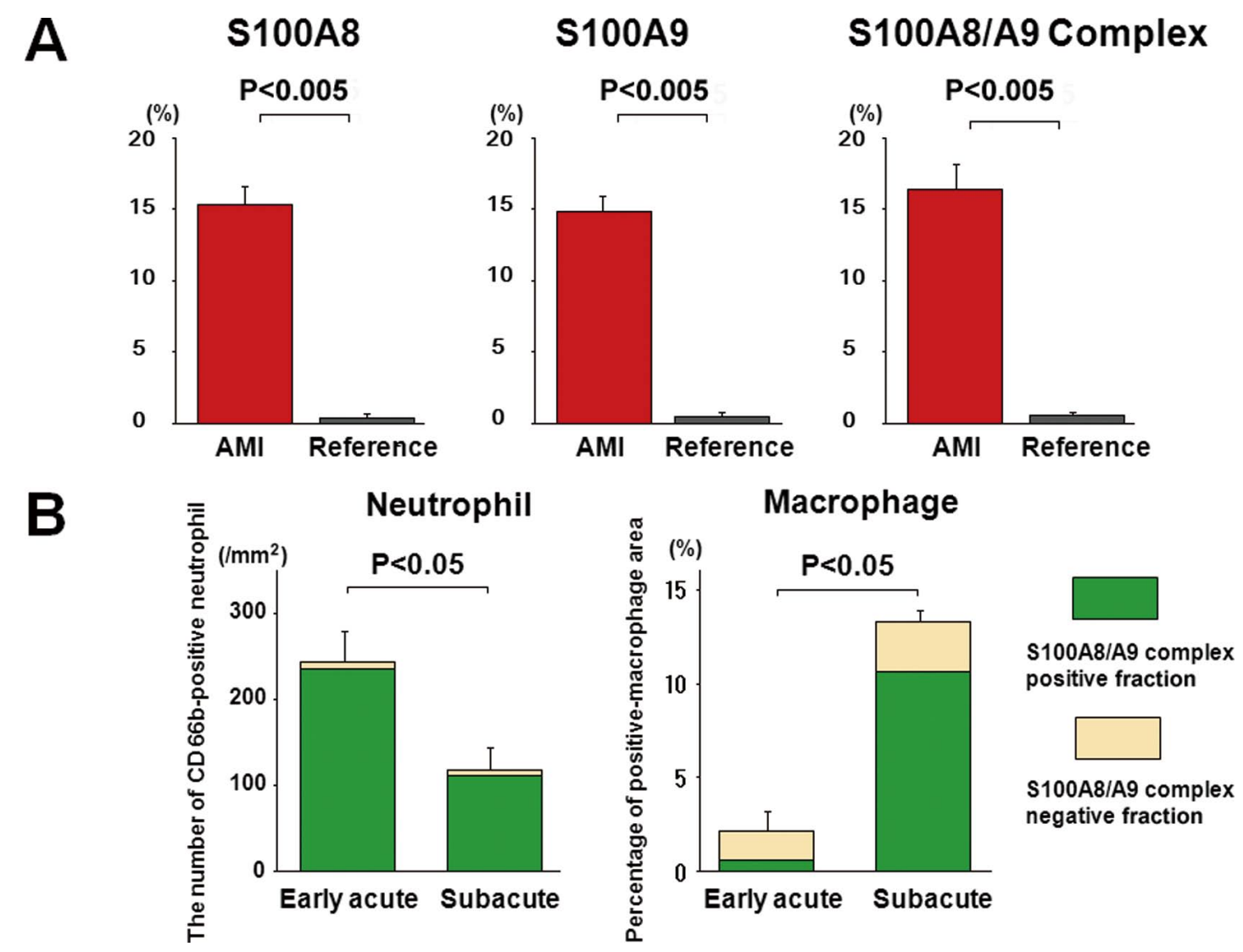

Figure 5. (A) Graphs showing the S100A8-, S100A9-, and S100A8/A9-complex-positive areas expressed as a percentage of the total surface area in autopsied human myocardium from AMI (red bars) and reference cases (grey bars). (B) Graphs showing the number of neutrophils $/ \mathrm{mm}^{2}$ and the macrophage-positive areas expressed as a percentage of the total surface in the infarcted myocardium in the early acute and subacute phases. Green indicates S100A8/A9-complex-positive fractions. AMI, acute myocardial infarction. 
cases (Figure 5A). In the infarcted myocardium of AMI patients, the number of CD66b-positive neutrophils was significantly higher $(\mathrm{P}<0.05)$ in the early acute phase than in the subacute phase. In contrast, the macrophage-positive area was significantly smaller $(\mathrm{P}<0.05)$ in the early acute phase than in the subacute phase (Figure 5B). In addition, the percentage of S100A8/A9-complex-positive macrophages in the total macrophage infiltration area was significantly higher

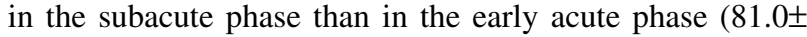
$3.5 \%$ vs $33.0 \pm 4.7 \%, \mathrm{P}<0.05)$. In contrast, there was no significant difference between the 2 groups in the percentage of S100A8/A9-complex-positive neutrophils in the total neutrophil number $(92.7 \pm 1.2 \%$ vs $96.3 \pm 1.7 \%, \mathrm{P}=\mathrm{NS}$ ) (Figure 5B).

\section{Discussion}

The novel findings of this study are as follows. First, in AMI patients who had undergone successful primary angioplasty, we showed a transient increase in serum levels of the S100A8/A9 complex with a peak value at 3-5 days after the onset of AMI. Second, we showed a distinct expression of the S100A8/A9 complex in neutrophils and macrophages infiltrating the infarct.

The source of the elevated serum level of the S100A8/A9 complex is attributable to inflammatory lesions in coronary atherosclerotic plaques, ${ }^{6,18}$ and in infarcted myocardium, because AMI is associated with both lesions. In the present study, to discriminate the source of the complex, we measured serum levels of the S100A8/A9 complex in patients with UAP, in whom the source of the complex may be limited to coronary vulnerable plaques. Although the serum level remained unchanged for 1 week in UAP patients, in AMI patients it increased by approximately 50\% at 3-5 days after the onset of AMI, and showed a still higher level at 6 8 days after the onset, as compared with the baseline value. These findings strongly suggest that serum S100A8/A9 is derived from neutrophils and macrophages infiltrating coronary atherosclerotic plaques and the infarcted area. In previous studies, ${ }^{6-8}$ blood concentrations of S100A8/A9 in AMI patients were measured only once, whereas we serially measured the serum levels after the onset of AMI, which lead us to assume the infarcted myocardium was an additional source of the complex.

As an alternate source of S100A8/9 in this setting, circulating leukocytes need to be considered. AMI initiates a systemic response that involves global activation of inflammatory systems. This global activation is partly evidenced by elevated CRP levels and elevated leukocyte counts. ${ }^{19,20}$ Thus, it is possible, as an alternate explanation, that the bulk of the circulating S100A8/A9 was derived from the increased numbers of activated circulating neutrophils and macrophages.

Our finding of statistically significant positive correlations between peak serum levels of the S100A8/A9 complex, and peak CK and CK-MB levels indicates that the S100A8/A9 level is largely influenced by infarct size. The level of complex also correlated with peak WBC and CRP levels, thus suggesting that the S100A8/A9 complex is heavily involved in inflammatory disorders. The role of the level of the S100A8/A9 complex in predicting the risk of cardiovascular events has been proposed, ${ }^{7,8}$ and it may serve as a biomarker of both the severity and prognosis of AMI.

In the present study, we demonstrated strong expression of the S100A8/A9 complex in the infarcted myocardium of AMI patients, thus suggesting that the S100A8/A9 complex, produced by activated neutrophils and macrophages, is involved in the pathophysiology of AMI. To the best of our knowledge, we are the first to describe the in situ localization of the S100A8/A9 complex in the infarcted myocardium of AMI patients. Regarding the staining pattern of S100A8, S100A9, and the S100A8/A9 complex in the myocardium, there was no significant difference among the 3 antibodies. It has previously been demonstrated in mice and humans that there is heterogeneity in the expression of S100A8, A9, and S100A8/9 complexes by regional macrophages in atherosclerosis. ${ }^{10,21}$ However, the expression profile of the monomers and the S100A8/9 complex by leukocytes in infarcted myocardium might be different from that in atherosclerosis.

In the AMI patients, there was a positive correlation between maximal serum levels of the S100A8/A9 complex and maximal blood neutrophil counts in the early acute phase. The circulating neutrophil count reflects activated neutrophils infiltrating the infarcted myocardium and which appear to be a source of S100A8/A9 complex production. Meanwhile, in the subacute phase, the source of S100A8/A9 complex production appeared to be the increased number of activated macrophages in the infarcted myocardium. The shift in the infiltrating inflammatory cells from neutrophils to activated macrophages may reflect the repair of the myocardium by granulation tissue. Enhanced expression of the S100A8/A9 complex in neutrophils and macrophages infiltrating the infarct area may be evidence of the role these phagocytes play in the pathophysiology of AMI. ${ }^{13}$ Elucidation of the functional role of the S100A8/A9 complex would aid in the development of future therapeutic strategies for AMI. Further investigations on the mechanistic role of the S100A8/A9 complex are necessary.

\section{Conclusion}

Neutrophils and macrophages infiltrating the infarcted myocardium are possibly responsible for the high serum levels of the S100A8/A9 complex found in AMI patients. S100A8/A9 complex may be an additional biomarker of the local inflammatory response following AMI.

\section{Study Limitations}

We suggest that the infarcted myocardium is an important source of S100A8/A9 based on prolonged elevation of S100A8/A9 levels following AMI and on the differences in the time course between AMI and UAP. Experiments assessing S100A8/A9 levels in the coronary sinus during coronary intervention and a comparison with peripheral blood levels would provide stronger support for this concept.

\section{Acknowledgment}

This study was supported in part by research grants from the Ministry of Health, Labor, and Welfare of Japan (Tokyo, Japan).

Conflict of interest: none declared.

\section{Disclosure}

\section{References}

1. Roth J, Vogl T, Sorg C, Sunderkotter C. Phagocyte-specific S100 proteins: A novel group of proinflammatory molecules. Trends Immunol 2003; 24: 155-158.

2. Rammes A, Roth J, Goebeler M, Klempt M, Hartmann M, Sorg C. Myeloid-related protein (MRP) 8 and MRP14, calcium-binding proteins of the $\mathrm{S} 100$ family, are secreted by activated monocytes via novel, tubulin-dependent pathway. J Biol Chem 1997; 272: 9496-9502. 
3. Frosch M, Vogl T, Seeliger S, Wulffraat N, Kuis W, Viemann D, et al. Expression of myeloid-related proteins 8 and 14 in systemiconset juvenile rheumatoid arthritis. Arthritis Rheum 2003; 48: $2622-2626$.

4. Ikemoto M, Tanaka T, Takai Y, Murayama H, Tanaka K, Fujita M. New ELISA system for myeloid-related protein complex (MRP8/14) and its clinical significance as a sensitive marker for inflammatory responses associated with transplant rejection. Clin Chem 2003; 49: $594-600$.

5. Miyamoto S, Ueda M, Ikemoto M, Naruko T, Itoh A, Tamaki S, et al. Increased serum levels and expression of S100A8/A9 complex in infiltrated neutrophils in atherosclerotic plaque of unstable angina. Heart 2008; 94: 1002-1007.

6. Altwegg LA, Neidhart M, Hersberger M, Müller S, Eberli FR, Corti R, et al. Myeloid-related protein 8/14 complex is released by monocytes and granulocytes at the site of coronary occlusion: A novel, early, and sensitive marker of acute coronary syndromes. Eur Heart J 2007; 28: 941-948.

7. Healy AM, Pickard MD, Pradhan AD, Wang Y, Chen Z, Croce K, et al. Platelet expression profiling and clinical validation of myeloidrelated protein-14 as a novel determinant of cardiovascular events. Circulation 2006; 113: 2278-2284.

8. Morrow DA, Wang Y, Croce K, Sakuma M, Sabatine MS, Gao H, et al. Myeloid-related protein 8/14 and the risk of cardiovascular death or myocardial infarction after an acute coronary syndrome in the Pravastatin or Atorvastatin Evaluation and Infection Therapy: Thrombolysis in Myocardial Infarction (PROVE IT-TIMI 22) trial. Am Heart J 2008; 155: 49-55.

9. McCormick MM, Rahimi F, Bobryshev YV, Gaus K, Zreiqat H, Cai $\mathrm{H}$, et al. S100A8 and S100A9 in human arterial wall: Implications for atherogenesis. J Biol Chem 2005; 280: 41521-41529.

10. Ionita MG, Vink A, Dijike IE, Laman JD, Peeters W, van der Kraak $\mathrm{PH}$, et al. High levels of myeloid-related protein 14 in human atherosclerotic plaques correlate with the characteristics of rupture-prone lesions. Arterioscler Thromb Vasc Biol 2009; 29: 1220-1227.

11. Croce K, Gao H, Wang Y, Mooroka T, Sakuma M, Shi C, et al.
Myeloid-related protein-8/14 is critical for the biological response to vascular injury. Circulation 2009; 120: 427-436.

12. Terasaki F, Fujita M, Shimomura H, Tsukada B, Otsuka K, Otsuka $\mathrm{K}$, et al. Enhanced expression of myeloid-related protein complex (MRP8/14) in macrophages and multinucleated giant cells in granulomas of patients with active cardiac sarcoidosis. Circ J 2007; 71: $1545-1550$

13. Frangogiannis NG, Smith CW, Entman ML. The inflammatory response in myocardial infarction. Cardiovasc Res 2002; 53: $31-$ 47.

14. Pathology of myocardial infarction. In: Virmani R, Burke A, Farb A, Atkinson JB. Cardiovascular pathology, 2nd edn. Philadelphia: WB Saunders, 2001; 155-178.

15. TIMI Study Group. The Thrombolysis In Myocardial Infarction (TIMI) trial: Phase I findings. $N$ Engl J Med 1985; 312: 932 - 936.

16. Braunwald E. Unstable angina: A classification. Circulation 1989; 80: $410-414$.

17. van der Loos CM, Becker AE, van den Oord JJ. Practical suggestions for successful immunoenzyme double-staining experiments. Histochem J 1993; 25: 1 - 13 .

18. Arakawa K, Yasuda S, Hao H, Kataoka Y, Morii I, Kasahara Y, et al. Significant association between neutrophil aggregation in aspirated thrombus and myocardial damage in patients with ST-segment elevation acute myocardial infarction. Circ J 2009; 73: 139-144.

19. O'Donoghue M, Morrow DA, Cannon CP, Guo W, Murphy SA, Gibson CM, et al. Association between baseline neutrophil count, clopidogrel therapy, and clinical and angiographic outcomes in patients with ST-elevation myocardial infarction receiving fibrinolytic therapy. Eur Heart J 2008; 29: 984-991.

20. Kruk M, Przyluski J, Kalinczuk L, Pregowski J, Deptuch T, Kadziela $\mathrm{J}$, et al. Association of non-specific inflammatory activation with early mortality in patients with ST-elevation acute coronary syndrome treated with primary angioplasty. Circ J 2008; 72: 205-211.

21. Eue I, Langer C, Eckardstein A, Sorg C. Myeloid related protein (MRP) 14 expressing monocytes infiltrate atherosclerotic lesions of ApoE null mice. Atherosclerosis 2000; 151: 593-597. 